\title{
Correction: The role of substance $P$ in epilepsy and seizure disorders
}

\section{Xue Feng Wang ${ }^{1}$, Tong Tong Ge ${ }^{1}$, Jie Fan ${ }^{1}$, Wei Yang ${ }^{1}$, Bingjin Li ${ }^{1}$ and Ran Ji Cui ${ }^{1}$}

${ }^{1}$ Jilin Provincial Key Laboratory on Molecular and Chemical Genetic, Second Hospital of Jilin University, Changchun, People's Republic of China

Correspondence to: Wei Yang, email: wyang2002@jlu.edu.cn

Bingjin Li, email: libingjin@jlu.edu.cn

Ran Ji Cui, email: cuiranji@jlu.edu.cn

Published: May 28, 2019

Copyright: Wang et al. This is an open-access article distributed under the terms of the Creative Commons Attribution License 3.0 (CC BY 3.0), which permits unrestricted use, distribution, and reproduction in any medium, provided the original author and source are credited.

This article has been corrected: The new Correspondence details are given below:

\section{Bingjin Li ${ }^{1}$}

Correspondence to: Bingjin Li, email: libingjin@jlu.edu.cn

Original article: Oncotarget. 2017; 8:78225-78233. https://doi.org/10.18632/oncotarget.20606 